\title{
Electronic transport properties of compounds with temperature unstable intermediate valence of $\mathrm{Ce}$
}

\author{
M.D.Koterlyn ${ }^{* 1,2}$, R.I.Yasnitskii ${ }^{1}$, B.S.Morokhivskii ${ }^{1}$ \\ 1 Ivan Franko National University of Lviv, \\ Dragomanova Str. 50, 79005 Lviv, Ukraine \\ 2 Academy of Bydgoszcz, Sq. Weyssenhoffa 11, 85-072 Bydgoszcz, Poland
}

Received March 12, 2004

\begin{abstract}
Results of a thermoelectric power component $\left(S_{f}\right)$ and electrical resistivity $\left(\rho_{f}\right)$ measurements connected with the temperature unstable intermediate valence of $\mathrm{Ce}$ are presented for $\mathrm{CeNi}, \mathrm{CeNi}_{2}$ and $\mathrm{CeNi}_{2} \mathrm{Si}_{2}$ compounds in the temperature range $4-800 \mathrm{~K}$. It is shown that dependences $S_{f}(T)$ and $\rho_{f}(T)$ are well described in a wide temperature range within the framework of a simple model of a narrow peak in the density of states $g_{f}(E)$ of Lorentzian shape near the Fermi level. The width of the peak depends on temperature and defines the characteristic single-ion Kondo temperature $T_{\mathrm{K}}$.
\end{abstract}

Key words: rare earth compounds, valence fluctuation, electrical resistivity, thermoelectric power

PACS: 72.20.Lp, 72.10.Fk, 75.30.Mb

\section{Introduction}

Metallic systems containing rare-earth elements with unstable $4 f$-electronic states have been very attractive for the last decades. A specific feature of this class of compounds turns out to be the anomalously high values of certain physical characteristics, e.g., the coefficient $\gamma$ of the electronic-specific heat, magnetic susceptibility $\chi$, thermoelectric power $S$, that can exceed the values typical of simple metals by $10-10^{3}$ times $[1,2]$. In the band energy representation, a formation of the resonance peaks of the density of $f$-states, $g_{f}(E)$, near the Fermi level $E_{\mathrm{F}}$ with a width of the order of the characteristic single-ion Kondo temperature $T_{\mathrm{K}}$ (the so-called Abrikosov-Suhl resonance [2]) corresponds to this phenomenon. Despite the presence of a large number of experimental and theoretical works devoted to the many-body

*E-mail: koterlyn@mail.lviv.ua 
effect studies when the conduction electrons interact with the $4 f$ electrons in the compounds of this class, the nature of the resonant structure of the $g_{f}(E)$ remains the main issue to be discussed in future. Of special interest herein there are the intermetallic compounds showing the temperature unstable intermediate valence (IV) of Ce. These are most suitable for a model description in a study of the formation mechanism of the $g_{f}(E)$ structure.

Transport properties of metallic compounds containing Ce with unstable $f$ electrons turn out to be most sensitive to the peculiarities of the near Fermi state spectrum and can be used as a "fine instrument" while studying the nature of the ground state. It is known that transport characteristics of such systems can be described in the relaxation time approximation with allowing for the exchange scattering of conduction electrons on local $f$-states [3-5]. However, with a detailed consideration of a number of important factors, such as the $f$-state orbital degeneracy, crystal field-split $f$-states, the $f$-state hybridization with the band electrons, many-body effects connected with $f$-electron correlation effects, one cannot succeed in obtaining a general solution of the transport coefficient behavior problem in a wide temperature range [6,7]. Moreover, one cannot succeed in coordinating the results of description of the transport coefficients with the data of measurements of other physical properties. All this contributed to the development of a number of phenomenological approaches to the description of transport properties of such systems [8-10]. However, detailed comparisons of calculation values of transport properties within the limits of approximations [8-10] with experimental ones in a wide temperature range have not been carried out yet. This caused an insufficient study of the possible usage of such experimental data for the determined resonance structure $g_{f}(E)$ parameters in a wide temperature range.

The given paper shows temperature dependences of the contribution of Ce $f$ states to the total thermoelectric power $\left(S_{f}\right)$ and to the electrical resistivity $\left(\rho_{f}\right)$ for compounds $\mathrm{CeNi}, \mathrm{CeNi}_{2}$ and $\mathrm{CeNi}_{2} \mathrm{Si}_{2}$ with different temperature stability IV of Ce. Based on the phenomenological approach offered by us earlier [10-12] there was carried out the analysis of $S_{f}(T), \rho_{f}(T)$ dependences as well as of the transformation of the resonance structure $g_{f}(E)$ near the Fermi level at temperature changes within the largest limits $(4-800 \mathrm{~K})$.

\section{Experimental data}

Preparation of polycrystalline samples and methods of the performed measurements were described in pervious papers $[13,14]$. Transport properties of the compounds $\mathrm{CeNi}, \mathrm{CeNi}_{2}, \mathrm{CeNi}_{2} \mathrm{Si}_{2}$ and the corresponding analogs with La were partially analysed by us earlier [13-16]. The thermoelectric power of compounds with Ce is positive and reveals a typical maximum. The electrical resistivity manifests essential discrepancies from the Bloch-Grüneisen law characteristic of the systems with IV of Ce. For compounds with La, the thermoelectric power and electrical resistivity behavior is similar to that observed in non-magnetic transition metals.

Assuming that the resistivity of the lanthanum-based counterpart can be taken 
as a good approximation of the phonon contribution to the total measured resistivity of $\mathrm{CeNi}, \mathrm{CeNi}_{2}$ and $\mathrm{CeNi}_{2} \mathrm{Si}_{2}$ we expressed $\rho_{\mathrm{Ce}}(T)$ for these compounds as follows:

$$
\rho_{\mathrm{Ce}}(T)=\rho_{0, \mathrm{Ce}}+\rho_{\mathrm{La}}(T)-\rho_{0, \mathrm{La}}+\rho_{f}(T),
$$

where $\rho_{0, \mathrm{Ce}}, \rho_{0, \mathrm{La}}$ and $\rho_{\mathrm{Ce}}, \rho_{\mathrm{La}}$ denote temperature-independent residual resistivity and total electrical resistivity of a compound with Ce and the corresponding isostructural analog with La. While analyzing the peculiarities of $\rho_{f}(T)$, the component $\rho_{0}$ can be neglected and $\rho_{f}(T) \approx \rho_{\mathrm{Ce}}(T)-\rho_{\mathrm{La}}(T)$ assumed.

When defining $f$-state contribution to the total thermoelectric power, the GorterNordheim relation $[10,11]$ is usually used

$$
S_{f}(T)=\frac{\left(S_{\mathrm{Ce}} \rho_{\mathrm{Ce}}-S_{\mathrm{La}} \rho_{\mathrm{La}}\right)}{\left(\rho_{\mathrm{Ce}}-\rho_{\mathrm{La}}\right)},
$$

where $S_{\mathrm{Ce}}, S_{\mathrm{La}}$ denote the total thermoelectric power of a compound with Ce and the corresponding isostructural analog with La. In our case we assumed that $S_{f}(T) \approx$ $S_{\mathrm{Ce}}(T)-S_{\mathrm{La}}(T)$. Such a ratio appears to be more convenient since the presence of the local structure $\mathrm{g}_{f}(E)$ near the level $E_{\mathrm{F}}$ is the domineering factor in forming the total thermoelectric power $\left(S_{\mathrm{Ce}}\right)$ and frequently it is not domineering in the case of the total electrical resistivity $\left(\rho_{\mathrm{Ce}}\right)$. It is particularly characteristic of Ce compounds with transition $3 d$-elements [13].

\section{Theoretical details}

Theoretically, the transport properties of the systems with the IV of Ce have been most logically studied by means of the Anderson model (AM) [5-7]. However, it has not been possible so far to derive analytical relationships allowing for a description of the behavior of the systems with transport properties using the Ce IV in a wide temperature range. This urged the development of various phenomenological approaches [8-13], based on a two-band model. According to [8-13], the main contribution to the transport properties was caused by scattering the electrons from a broad $s(d)$-band to a narrow $f$-band of Lorentzian shape. The scattering process can be described by the relaxation time in the form of

$$
\tau_{\mathrm{sf}}(E, T)^{-1} \propto g_{f}(E) R_{\mathrm{ph}}(T) .
$$

The function $R_{\mathrm{ph}}(T)$ takes into account the possible phonon-induced interband $s-f$ transition.

From the linearized Boltzmann transport equation, the following expressions for electrical resistivity component and thermoelectric power connected with the IV of Ce have been

$$
\begin{array}{rlrl}
\rho_{f}(T) & =\frac{1}{e^{2}} \frac{1}{L_{0}}, & & S_{f}(T)=-\frac{1}{|e|} \frac{L_{1}}{L_{0}}, \\
L_{n} & =\int_{-\infty}^{\infty} E^{n} \tau_{\mathrm{sf}}(E, T)\left(-\frac{\partial f}{\partial E}\right) \mathrm{d} E, & n=0,1
\end{array}
$$


where $f(E)$ is the Fermi-Dirac distribution function. The contribution of such $s$ - $f$ transitions to thermoelectric power in the approximation of an independent scattering on $f$-centers can be written as $[13,17]$

$$
S_{f}(T)=\frac{2}{3} \pi^{2} \frac{k_{\mathrm{B}}}{|e|} \frac{T \varepsilon_{f}}{\left(\pi^{2} / 3\right) T^{2}+\varepsilon_{f}^{2}+\Gamma_{f}^{2}},
$$

where $\varepsilon_{f}$ and $\Gamma_{f}$ are the position of the $g_{f}(E)$ peak relative to the Fermi level $E_{\mathrm{F}}$ and its width, respectively. Parameter $k_{\mathrm{B}}$ is the Boltzmann constant. Equation (1) satisfactorily describes the temperature dependences of thermoelectric power of the majority of systems with IV of Ce even without taking into account the possible temperature dependences of the parameters $\varepsilon_{f}$ and $\Gamma_{f}$. However, the parameters of the $g_{f}(E)$ peak determined from equation (1) do not quite agree with those determined based on the measurements of thermodynamic and spectroscopic characteristics $[2,18,19]$. The relationship between the parameters $\varepsilon_{f}$ and $\Gamma_{f}$ considerably deviates from that assumed in the AM [6] or in the local Fermi-liquid model [20] (by the thermopower data $\varepsilon_{f} / \Gamma_{f}<1[8,9,13,17]$ when theoretical $\varepsilon_{f} / \Gamma_{f}>1$ ). In the case of strong orbital degeneracy of $f$-states $\left(N_{f} \gg 1\right)$, which occurs in systems with intermediate Ce valence, it is appropriate to use the well-known relation connecting the parameters $\varepsilon_{f}, \Gamma_{f}$ and $T_{\mathrm{K}}\left(\varepsilon_{f} \approx T_{\mathrm{K}}\right.$ and $\left.\Gamma_{f} \approx \pi T_{\mathrm{K}} / N_{f}[6]\right)$. Then, in place of equation (1), we obtain a more convenient expression,

$$
S_{f}(T)=\frac{2}{3} \pi^{2} \frac{k_{\mathrm{B}}}{|e|} \frac{T \cdot T_{\mathrm{K}}}{\left(\pi^{2} / 3\right) T^{2}+\left(1+\pi^{2} / N_{f}^{2}\right) T_{\mathrm{K}}^{2}},
$$

with only one fitting parameter $\mathrm{T}_{\mathrm{K}}$.

Recently we have shown [12] that a good agreement of the thermoelectric power curves calculated by equation (2) with the experimental data can be obtained only when the temperature dependence of the parameter $T_{\mathrm{K}}$ is taken into account. This especially holds true for systems with the IV states of Ce, which are sensitive to temperature. There arises an important problem of realistic estimations of the temperature behavior of $T_{\mathrm{K}}$ and their possible usage when describing electrical resistivity within the framework of the accepted model.

With the aim of getting some idea of the behavior of the calculation $\rho_{f}(T)$, in order to describe the contribution of phonon-induced interband $s$ - $f$ transition to the resistance we use the simplest two-band spherical Mott-Wilson model [21] widely employed in nonmagnetic transition metals with an incomplete $d$ shell. Taking into consideration only the $N$-process and temperature independent values of a matrix element for phonon induced $s$ - $f$ transition, we receive

$$
\begin{aligned}
\rho_{f}(T) & =\frac{R_{0} \cdot R_{\mathrm{ph}}(T)}{\left(\pi^{2} / 3\right) T^{2}+\left(1+\pi^{2} / N_{f}^{2}\right) T_{\mathrm{K}}^{2}}, \\
R_{\mathrm{ph}}(T) & =n_{s}(T)^{-1}\left(\frac{T}{\theta_{\mathrm{D}}}\right)^{3} J_{3}\left(\frac{\theta_{\mathrm{D}}}{T}\right),
\end{aligned}
$$


where $\theta_{\mathrm{D}}$ is the Debye temperature, $R_{0}$ is the coefficient taking into account a number of characteristic parameters of the model [21], $n_{s}(T)$ is the effective number of $s$ electrons per atom depending on the temperature caused by the changes of $f$ state occupation. The functions $J_{3}(x)$ are the well-known transport integrals defined by

$$
J_{3}(x)=\int_{0}^{x} \frac{y^{3} \mathrm{~d} y}{\left(e^{y}-1\right)\left(1-e^{-y}\right)} .
$$

Due to the special sensitivity of thermoelectric power to the parameters of the fine structure density of states, a detailed concordance of the calculation with the experiment was carried out only for the $S_{f}(T)$ component. The values of $S_{f}$ calculated by equation (2) were normalized by the coefficient $p=S_{f \max , \exp } / S_{f \max , \text { calc }}$, where $S_{f \text { max,exp }}$ and $S_{f \text { max,calc }}$ denote the maximal values of the experimental thermoelectric power and the calculated one. The parameter $p$ takes into account the discrepancy between the experimental values of $S_{f}(T)$ and those calculated without allowing in (2) for the concentration of scattering $f$-centers and the presence of other scattering mechanisms in the compound under study [22]. The dependences $T_{\mathrm{K}}(T)$, obtained due to such a concordance, were used for the estimation of $\rho_{f}(T)$ and some other crystal physical characteristics.

\section{Results and analysis}

According to spectroscopic measurements [23], transition of Ce from a state with strongly mixed valence $\left(\mathrm{Ce}^{3.2+}\right)$ to almost magnetic one $\left(\mathrm{Ce}^{3+}\right)$ is observed in $\mathrm{CeNi}$ in the temperature range $T<300 \mathrm{~K}$. The similar decrease of the $\mathrm{Ce}$ valence $(\approx 0.1-0.2)$ takes place in the case of $\mathrm{CeNi}_{2}$ and $\mathrm{CeNi}_{2} \mathrm{Si}_{2}$ reaching the state of $\mathrm{Ce}^{3.1+}[23,24]$. Temperature dependences of the $f$-states of Ce contribution to the total thermoelectric power $\left(S_{f}\right)$ for compounds $\mathrm{CeNi}, \mathrm{CeNi}_{2}$ and $\mathrm{CeNi}_{2} \mathrm{Si}_{2}$ are presented in figure 1. Apparently, typical features of the $S_{f}(T)$ dependence for the compounds in question appear to be the presence of asymmetric maximum (qualitatively $S_{f} \sim T$ at $T<T_{S_{f \max }}$ and $S_{f} \sim T^{-1}$ at $T>T_{S_{f \max }}$ ) and a certain deviation from linear behavior $S_{f} \sim T$ at low $T$ with the occurrence of positive curvature. A good agreement of the calculated thermoelectric power (figure 1, solid curve) with the experiment is reached when the temperature dependence of the parameter $T_{\mathrm{K}}$ is considered (figure 2$)$. In the low temperature range $\left(T<T_{S_{f \max }}\right.$ ), the parameter $T_{\mathrm{K}}$ sharply increases until values $T_{\mathrm{K}} \approx(2-5) T_{S_{f \max }}$. Linearity of the dependence $S_{f}(T)$ at $T<T_{S_{f \max }}$ frequently observed in such systems $[2,8,13]$ is achieved only at $T_{\mathrm{K}}=$ const according to equation (2). Such a dependence is shown for $\mathrm{CeNi}_{2} \mathrm{Si}_{2}$ (figure 1, dotted curve calculated at $T_{\mathrm{K}}=680 \mathrm{~K}$ ). The appearance of the positive curvature on the dependence of $S_{f}(T)$ in the low temperature range and the corresponding increase of $T_{\mathrm{K}}$ (figure 2) makes it possible to coordinate the parameters of the resonant structure $g_{f}(E)$ with the main thermodynamic properties of crystals. 


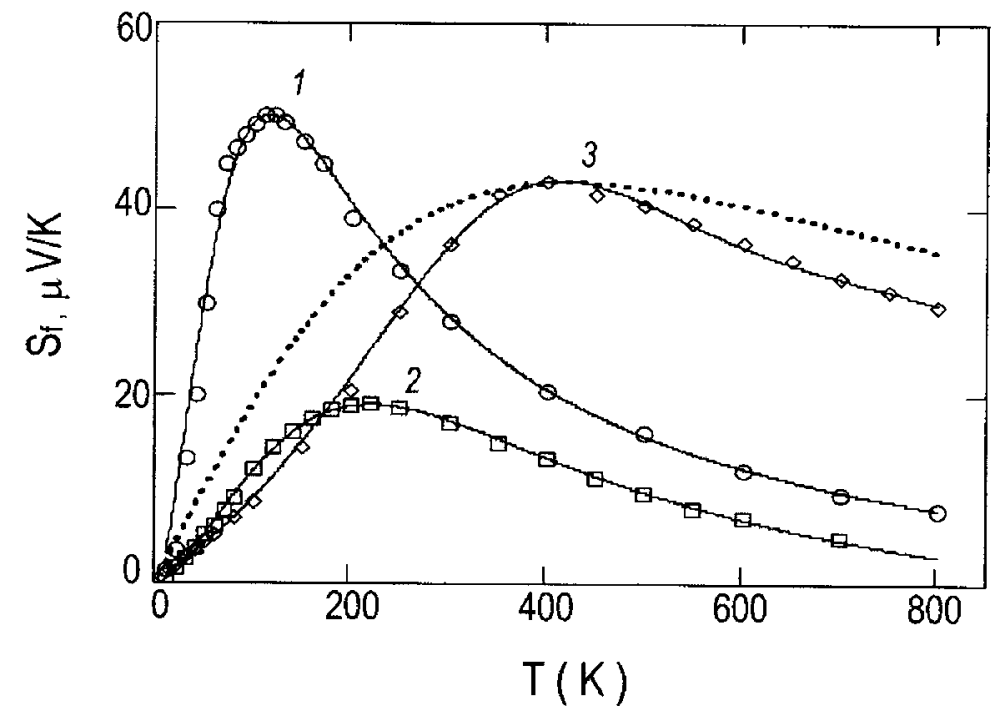

Figure 1. Temperature dependences of the contribution of Ce $f$-states to the total thermopower of $\mathrm{CeNi}(1), \mathrm{CeNi}_{2}$ (2) and $\mathrm{CeNi}_{2} \mathrm{Si}_{2}$ (3). Solid lines plot thermopower calculated from equation (2) in the suggested temperature dependence of parameter $T_{\mathrm{K}}$ and $T_{\mathrm{K}}=$ const (dotted curve).

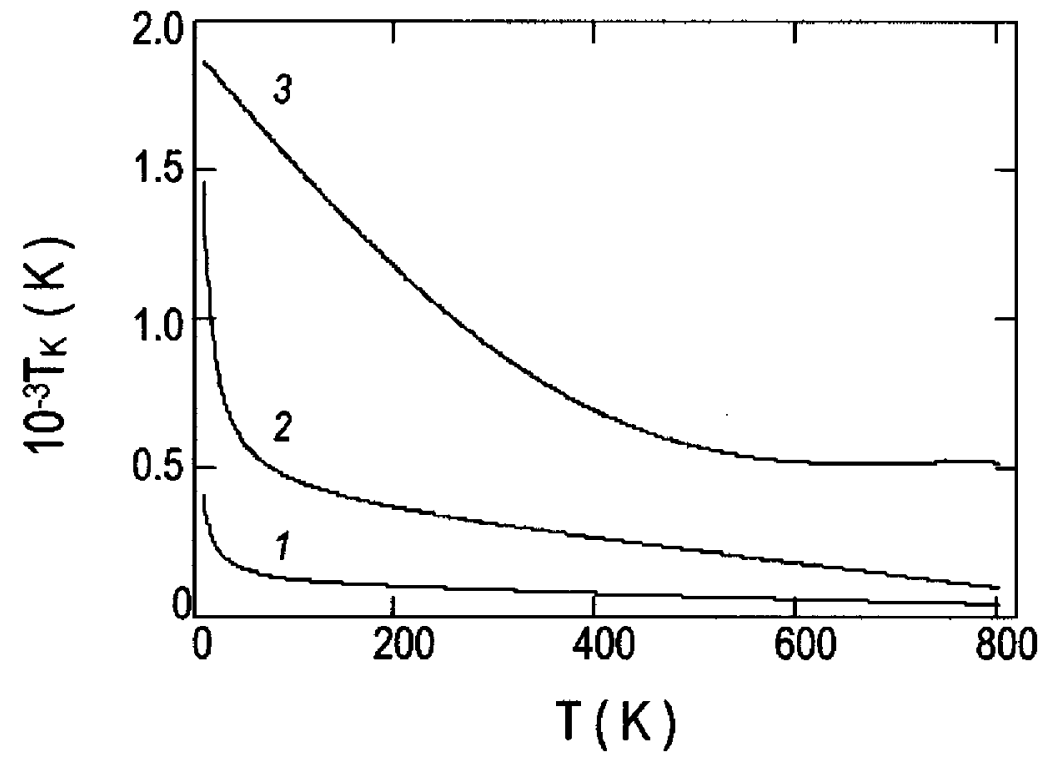

Figure 2. Temperature dependences of the parameter $T_{\mathrm{K}}$ of $\mathrm{CeNi}(1), \mathrm{CeNi}_{2}(2)$ and $\mathrm{CeNi}_{2} \mathrm{Si}_{2}$ (3), obtained based on the agreement of experimental thermoelectric power with that calculated by equation (2). 
It is well known $[6,25]$ that the majority of thermodynamic properties of the systems with the IV of Ce are satisfactorily described within the framework of a single impurity Anderson model with allowing for strong orbital degeneration

$$
\chi(0)=\frac{\mu^{2} n_{f}(0)}{3 T_{\mathrm{K}}(0)}
$$

and

$$
\gamma(0)=\frac{\pi^{2} k_{\mathrm{B}}^{2} n_{f}(0)}{3 T_{\mathrm{K}}(0)}
$$

where $\mu^{2}=g_{J}^{2} J(J+1) \mu_{\mathrm{B}}^{2}$ is the effective magnetic moment of the cerium $4 f^{1}$ state, $n_{f}(0)$ and $T_{\mathrm{K}}(0)$ is the electronic occupation of $f$-states of Ce and singleion Kondo temperature, respectively, at low temperatures. To assess the values of $T_{\mathrm{K}}(0)$ we use the known data [23] for the occupation of $f$-states of $\mathrm{Ce}$ in $\mathrm{CeNi}$ $\left(n_{f}(0)=0.88\right)$ determined at $T=80 \mathrm{~K}$. To deduct the contributions of $d$-states of Ni to the values of coefficients $\gamma(0)$ and $\chi(0)$, it is possible to use the similar data for LaNi. According to the data $[26,27], \gamma(0)=65 \mathrm{~mJ} \cdot \mathrm{mol}^{-1}(\mathrm{CeNi}), 5 \mathrm{~mJ} \cdot \mathrm{mol}^{-1}(\mathrm{LaNi})$; $\chi(0)=2.2 \cdot 10^{-3} \mathrm{emu} \cdot \mathrm{mol}^{-1}(\mathrm{CeNi}), 0.2 \cdot 10^{-3} \mathrm{emu} \cdot \mathrm{mol}^{-1}(\mathrm{LaNi})$. After substitution of the data presented in equations (5) and (6), we obtain $T_{\mathrm{K}}(0)=350$ and $390 \mathrm{~K}$, correspondingly. Results of the estimation of $T_{\mathrm{K}}(0)$ are in a good agreement with those determined by means of the thermoelectric power at low temperatures (we obtain $T_{\mathrm{K}}(0)=360 \mathrm{~K}$ at $\left.T=15 \mathrm{~K}\right)$. A similar assessment carried out for $\mathrm{CeNi}_{2}$ in [19] gives $T_{\mathrm{K}}(0)=1500 \mathrm{~K}$, which is also in a fair agreement with our calculations (we obtain $T_{\mathrm{K}}(0)=1450 \mathrm{~K}$ by the thermoelectric power data at $T=15 \mathrm{~K}$ ).

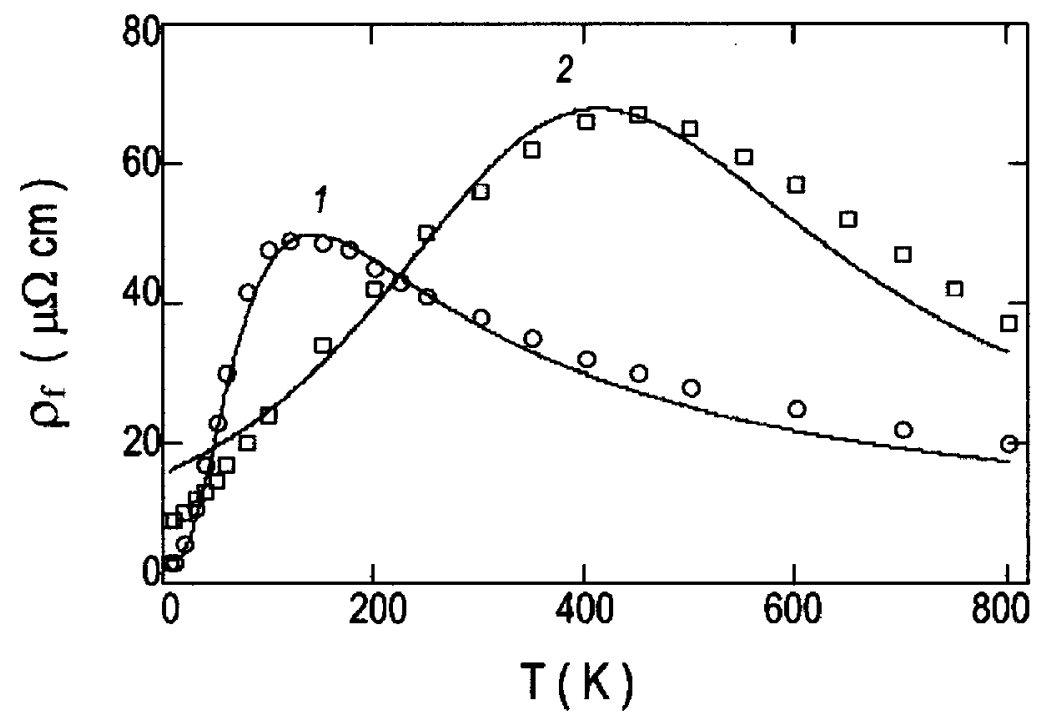

Figure 3. Temperature dependences of the contribution of $\mathrm{Ce} f$-states to the total electrical resistivity of $\mathrm{CeNi}(1)$ and $\mathrm{CeNi}_{2} \mathrm{Si}_{2}$ (2). Lines plot calculated dependences according to equation (3). 
The experimental and calculated (solid curves) dependences of the component $\rho_{f}(T)$ are shown in figure 3. The component for $\mathrm{CeNi}_{2}$ is not given, $\mathrm{LaNi}_{2}$ being an unsuitable analogue for singling out a phonon component of electrical resistivity. Calculation of normalized resistivity $\rho_{f}(T) / R_{0}$ was carried out by equation (3) with the use of the found dependence $T_{\mathrm{K}}(T)$ and additionally taking into account the residual electrical resistivity $\rho_{f}(0)$, taken from the experiment. The coefficient $R_{0}$ is chosen such that the numerical value of $\rho_{f \max } / R_{0}$ agrees with the experimental data. In the case of $\mathrm{CeNi}$, the best agreement between the calculation and the experiment is reached when the function $R_{\mathrm{ph}}(T)$ in equation (3) has the form of equation (4) with $\theta_{D}=110 \mathrm{~K}$ and dependence $n_{s}(T)=n_{s}(0)+0.7 \cdot 10^{-4} T-0.5 \cdot 10^{-2} T^{1 / 2}$, found with the spectroscopic data [23]. In case of $\mathrm{CeNi}_{2} \mathrm{Si}_{2}$, the best concordance with the experiment is achieved only assuming $R_{\mathrm{ph}}(T)=$ const. The necessity of taking into account the temperature dependence of the time relaxation testifies to a considerable effect of electron-phonon interaction in $\mathrm{CeNi}$ on $\rho_{f}(T)$ behavior. This fact agrees with an anomalously strong interaction of the electron system with lattice vibrations revealed previously [28]. In the case of $\mathrm{CeNi}_{2} \mathrm{Si}_{2}$, the behavior type of $\rho_{f}(T)$ corresponds to the Mott scattering on $s$ - $f$-transitions with allowing for the temperature narrowing of the peak density of states $g_{f}(E)$. Some discrepancy of the calculation with the experiment for $\mathrm{CeNi}_{2} \mathrm{Si}_{2}$ in the low temperature range can be caused by partial display of coherent effects.

\section{Conclusions}

By the set of the data presented one can conclude that the transport properties of the compounds with the temperature unstable IV of Ce can be satisfactorily described in a wide temperature range within the framework of a simple model of a narrow peak of the density of states $g_{f}(E)$ of Lorentzian shape with allowing for its narrowing caused by stabilization of the $\mathrm{Ce}^{3+}$ states as the temperature increases. The values of $T_{\mathrm{K}}$ obtained at a low temperature are completely realistic and in a good agreement with the assessments made by other methods. The differences in the character of temperature dependences of electrical resistivity for $\mathrm{CeNi}$ and $\mathrm{CeNi}_{2} \mathrm{Si}_{2}$ are probably caused by different roles of band states played at forming IV of Ce.

\section{References}

1. Stewart G.R. // Rev. Mod. Phys., 1984, vol. 56, p. 755.

2. Brandt N.B., Moshchalkov V.V. // Adv. Phys., 1984, vol. 33, p. 373.

3. Cornut D., Coqblin B. // Phys. Rev. B, 1972, vol. 5, p. 4541.

4. Bhattacharjee A.K., Coqblin B. // Phys. Rev. B, 1976, vol. 13, p. 3441.

5. Fischer K.H. // Z. Phys. B. - Cond. Matt., 1989, vol. 76, p. 315.

6. Bickers N.E., Cox D.L., Wilkins J.W. // Phys. Rev. B, 1987, vol. 36, p. 2036.

7. Zlatic V., Horvatic B., Milat I., Coqblin B., Czycholl G., Grenzebach C. // Phys. Rev. B, 2003, vol. 68, p. 104432. 
8. Gottwick U., Gloos K., Horn S., Steglich F., Grewe N. // J. Magn. Magn. Mater., 1985 , vol. 47,48 , p. 536.

9. Garde G.S., Ray J. // Phys. Rev. B, 1995, vol. 51, p. 2960.

10. Koterlyn M.D., Babych O.I., Koterlyn G.M. // J. Alloys Comp., 2003, vol. 348, p. 52.

11. Koterlyn M.D., Babych O.I., Yasnitskii R.I. // Fiz. Tverd. Tela (St. Petersburg), 2002, vol. 44, p. 792 (in Russian).

12. Koterlyn M.D., Yasnitskii R.I., Koterlyn G.M., Morokhivskii B.S. // J. Alloys Comp., 2003, vol. 348, p. 52.

13. Koterlyn M.D., Babych O.I., Lutsiv R.V., Nemoshkalenko V.V., Nikolajev L.I., Jushchenko A.V. Preprint, No. 11, IMF, Kijev, 1986.

14. Lutsiv R.V., Koterlyn M.D., Babych O.I. // Fiz. Tverd. Tela (Leningrad), 1984, vol. 26, p. 1781 (in Russian).

15. Levin E.M., Lutsiv R.V., Finkelshtein L.D., Samsonova N.D., Yasnitskii R.I. // Fiz. Tverd. Tela (Leningrad), 1981, vol. 23, p. 2401 (in Russian).

16. Koterlyn M.D., Lutsiv R.V., Yasnitskii R.I. // Ukr. Fiz. Jurn., 1984, vol. 29, p. 1587 (in Russian).

17. Koterlyn M.D., Lutsiv R.V. Physics and Chemistry of Rare-Earth Semiconductors. Novosibirsk, Nauka, 1990, p. 18.

18. Murani A.P., Raphel R., Bowden Z.A., Eccleston R.S. // Phys. Rev. B, 1996, vol. 53, p. 8188 .

19. Murani A.P., Eccleston R.S. // Phys. Rev. B, 1996, vol. 53, p. 48.

20. Newns D.M., Newson A.C. // J. Phys. F: Metal Phys., 1980, vol. 10, p. 2429.

21. Colquitt L. // J. Appl. Phys., 1965, vol. 36, p. 2454.

22. Lutsiv R.V., Koterlyn M.D. // Fiz. Tverd. Tela (Leningrad), 1983, vol. 25, p. 2484 (in Russian).

23. Sovestnov A.E., Shaburov V.A., Markova J.A., Savitskii E.M., Chistjakov O.D., Shkatova T.M. // Fiz. Tverd. Tela (Leningrad), 1981, vol. 23, p. 2827 (in Russian).

24. Groshev M.N., Koterlyn M.D., Levin E.M., Lutsiv R.V., Miftahov N.M., Smirnov Yu.P., Sovestnov A.E., Tyunis A.V., Shaburov V.A., Yasnitskii R.I., Kuzmina S.M., Petrova V.I., Tyukavin V.A. // Fiz. Tverd. Tela (Leningrad), 1986, vol. 28, p. 2711 (in Russian).

25. Ramakrishnan T.V., Sur K. // Phys. Rev. B, 1982, vol. 26, p. 1798.

26. Gignoux D., Givord F., Lemaire R., Tasset F. // J. Less-Common. Met., 1983, vol. 94, p. 165.

27. Isikawa Y., Mori K., Fujii A., Sato K. // J. Phys. Soc. Jap., 1986, vol. 55, p. 3165.

28. Clementyev E.S., Braden M., Lasukov V.N., Alekseev P.A., Mignot J.-M., Sadikov I.P., Hiess A., Lapertot G. // Physica B: Cond. Matter., 1999, vol. 259-261, p. 42. 


\section{Електронні транспортні властивості сполук 3 температурно-нестабільною проміжною валентністю Ce}

\section{М.Д.Котерлин ${ }^{1,2}$, Р.Й.Ясницький ${ }^{1}$, Б.С.Морохівський ${ }^{1}$}

1 Львівський національний університет імені Івана Франка, вул. Драгоманова 50, 79-005, Львів, Україна

2 Академія Бидгощска, пл. Вейссенгоффа 11, 85-072 Бидгощ, Польща

Отримано 12 березня 2004 р.

Для сполук $\mathrm{CeNi}, \mathrm{CeNi}_{2}$ та $\mathrm{CeNi}_{2} \mathrm{Si}_{2}$ представлені результати вимірювання в інтервалі температур 4-800 К складових термо-е.р.с. $\left(S_{f}\right)$ та електроопору $\left(\rho_{f}\right)$, викликаних температурно-нестабільною проміжною валентністю Се. Показано, що залежності $S_{f}(T)$ та $\rho_{f}(T)$ в широкому інтервалі температур добре описуються на основі простої моделі вузького піку густини станів $g_{f}(E)$ лоренцівської форми біля рівня Фермі. Ширина піку залежить від температури і визначається одно-іонною температурою Кондо $T_{\mathrm{K}}$.

Ключові слова: рідкісноземельні сполуки, валентні флуктуації, електроопір, термоелектрорушійна сила

PACS: 72.20.Lp, 72.10. Fk, 72.15.Qm, 75.30.Mb 